\title{
Prevalence and associated factors with depression and anxiety in prisoners in South of Brazil
}

\author{
Caroline Ribeiro Costa ${ }^{1}$ \\ https://orcid.org/0000-0003-0889-8030 \\ RAÚL ANDRÉS MENDOZA SASSI ${ }^{1}$
}

Vinícius de Souza TÍmbola ${ }^{1}$

https://orcid.org/0000-0001-6851-8445

TALITA RUBIN LAZZARI ${ }^{1}$

https://orcid.org/0000-0002-7262-7027

Ana Julia Reis ${ }^{1}$

https://orcid.org/0000-0002-0317-9179

CARLA VITOLA GONCCALVES1

1 Graduate Program in Health Sciences, Federal University of Rio Grande (FURG), Rio Grande, RS, Brazil.

Received: 09/13/2018 - Accepted: 04/15/2020

DOl: 10.1590/0101-60830000000239

\begin{abstract}
Background: Brazil has the third largest prison population in the world. Research in the world has confirmed a high prevalence of mental disorders in this population. Objective: To identify prevalence and associated factors with depression and anxiety in prisoners of the closed prison system. Methods: This is a cross-sectional study with 643 prisoners were interviewed in six prisons in Rio Grande do Sul, Brazil. To evaluate depression and anxiety, the Mini International Neuropsychiatric Interview 5.0 (MINI) was used and sociodemographic, inprisonment and lifestyle habits variables were also collected. Results: The prevalence of depression found in the study was 20.6\% (95\% CI: 17.5-23.8) and of anxiety was 19.9\% (95\% CI: 16.8-23.0). The following were identified as risk factors for depression: being female, having a history of mental illness, non-white skin color, having a religion, not receiving visits, smoking, using drugs and not performing physical activities. Risk factors for anxiety were: being female, having a history of mental illness, a family history of mental illness, smoking and using drugs. Discussion: The study confirmed the high rates of depression and anxiety in the population deprived of liberty. In addition, women were twice as likely to have both disorders compared to men.
\end{abstract}

Costa CR et al. / Arch Clin Psychiatry. 2020;47(4):89-94

Keywords: Epidemiology, depression, anxiety, prisoners, MINI.

\section{Introduction}

The health of the population deprived of liberty has been a frequent subject of study, as more than 10 million people are in prison worldwide $^{1}$. Brazil has the third largest prison population in the world, with nearly 726,000 persons deprived of their liberty ${ }^{2}$. Research on mental health of prisoners in the world confirmed a high prevalence of mental disorders in this population, which ranges from $25 \%$ to $60 \%{ }^{3-5}$ and can reach up to $80 \%^{6}$.

Major Depressive Episode is characterized primarily by depressed mood and loss of interest or pleasure for at least two weeks. Other symptoms are significant weight loss or gain, insomnia or hypersomnia, restlessness or psychomotor retardation, fatigue, feelings of worthlessness or guilt, decreased concentration and attention, recurrent thoughts about death, suicidal ideation or attempted suicide? . For didactic purposes in this article, we will use the term "depression" as a synonym for Major Depressive Episode.

According to the World Health Organization (WHO), the prevalence of depression in the world population is $4.4 \%$ and in Brazil, it is $5.8 \%{ }^{8}$. According to the Brazilian Institute of Geography and Statistics (IBGE) ${ }^{9}$, the prevalence of people diagnosed with depression in the state of Rio Grande do Sul is of $13.2 \%$. In a study with 3,391 low-income residents of Rio Grande do Sul ${ }^{10}$, the prevalence of depression was $16.1 \%$. Although there is no consent on the prevalence of depression among prisoners, the number varies between 16.7 and $72.6 \%$ among men ${ }^{11-14}$, and among women it ranges from 13 to $80 \%$, surpassing the rates of the general population $6,15,16$. The wide range of estimates found in literature can be because of the use of different instruments for the diagnosis of mental disorders. The main factors associated with depression in the population deprived of liberty were chronic lack of physical health, physical attacks, having children, regular or poor attachment to family members ${ }^{17}$ and having been sexually victimized at some point in life ${ }^{15}$. Receiving recent visitors was identified as the main protective factor ${ }^{14,18,19}$.

Another frequent disturb among prisoners is Generalized Anxiety Disorder, which is characterized by anxiety and excessive worries occurring on most days for at least six months. Other common symptoms are restlessness, fatigability, difficulty concentrating, irritability, muscle tension and sleep disturbances ${ }^{7}$. According to the $\mathrm{WHO}$, the prevalence of people with anxiety in the general population is of $3.4 \% \%^{8}$, whereas in the imprisoned population it ranges from $8.3 \%$ to $77.8 \% 12,13,20-22$. The main factors associated with anxiety among prisoners were being a smoker ${ }^{19}$, being arrested for the first time ${ }^{23}$ and a having history of domestic violence in childhood ${ }^{12}$.

In Brazil, there are still few studies on the mental health of the population deprived of liberty. It is important to understand the panorama of mental health in this context so that effective actions can be articulated aiming to minimize factors that can aggravate or trigger mental disorders. The present study aimed to identify the prevalence of depression and anxiety and their associated factors among the prison population in Southern Brazil. 


\section{Methods}

This work is part of a larger project entitled "Prison Health", which aimed to evaluate the health of the prison population concerning mental health, nutritional health, tuberculosis and knowledge and/ or diagnosis of sexually transmitted infections (STIs). The study was performed from May 2017 to January 2018 in the 5th Regional Penitentiary Station - South Region (5th RPS), which comprises the State Prisons of Camaquã, Pelotas, Jaguarão, Canguçu, Rio Grande and Santa Vitória do Palmar, and consisted of a cross-sectional study with random sample stratified by prison.

When we started the survey, the prison population in closed system in the $5^{\text {th }}$ RPS was of 1,407 men and 100 women. The eligible population for the study consisted of all men and women who at the time of the interview were serving their sentences under a closed regime in regional prisons of Southern Brazil. Prisoners who had some cognitive impairment that prevented them from understanding the questions were excluded.

Due to the wide prevalence range found in the scientific literature, a first calculation of sample size was performed using all mean values found. Subsequently, a new sample calculation was performed based on the prevalence detected in a pilot study, which was performed with 35 prisoners from the semi-open system of the Rio Grande Prison. We used a margin of error of 5\%, a 95\% confidence interval and $10 \%$ were added for losses and refusals. For depression, using a prevalence of $23 \%$, the $\mathrm{N}$ found was of 376 inmates, while for anxiety, using a prevalence of $17 \%$, the $\mathrm{N}$ found was of 310 inmates.

For the calculation of associated factors, we also used the prevalence rates found in the pilot study, 95\% confidence interval, relative risk of 2.0 and statistical power of $80 \%$, adding $10 \%$ for losses and $40 \%$ for confounding factors. After calculating the associated factors we opted for the largest $\mathrm{N}$ found, that was of 755 inmates.

For the male inmates we performed a stratified random sample proportional to population size and logistics of each prison. This sampling process was based was based on lists provided by each prison. A "leap of three" was used for the selection of the prisoners to be interviewed. The expression "leap of three" means that the first name was randomly chosen from a list, and the following names were always selected with an interval of three individuals until completing the $\mathrm{N}$ defined in that prison. All imprisoned women were included.

A total of 643 (77\%) inmates participated in the study, distributed as follows: Rio Grande (243), Pelotas (233), Camaquã (93), Canguçu (29), Jaguarão (23) and Santa Vitória do Palmar (22). There were $188(22.5 \%)$ refusals and four (0.5\%) losses during the interviews.

Data collection took place according to the structure, logistics and security of each prison, and it was carried out in classrooms, outpatient clinics and in galleries' corridors, most of them occurring behind bars. Each inmate was individually informed about the research and later presented to the Informed Consent Form (ICF).

Interviews were conducted using a pre-coded questionnaire containing sociodemographic variables (age, skin color, marital status, education and income), prison situation (time of penalty, type of crime and occurrence of visitations), mental health (family and personal history of mental health), life habits (religion, physical activity and use of tobacco, alcohol and other drugs). For the evaluation of mental disorders, we used the modules Major Depressive Episode and Generalized Anxiety Disorder of the Mini International Neuropsychiatric Interview (MINI Plus), validated for the Brazilian population ${ }^{21}$. MINI Plus is a standardized diagnostic clinical interview with diagnostic criteria of the Diagnostic and Statistical Manual of Mental Disorders (DSM-V) and the International Statistical Classification of Diseases (ICD-10). All interviewers were trained to apply this instrument accordingly.

Data was codified and later double typed from the questionnaires in a database created in the software Epidata 3.1. Data analysis was performed using STATA 14.0. For the bivariate analysis of the sample, Prevalence Ratio (PR), 95\% confidence intervals and Pearson's chisquare test were calculated, adopting a p-value $<0.05$ of a two-tailed test. Adjusted analysis was performed using Poisson regression according to a 3-level hierarchy model for causal effects ${ }^{24}$. The first and most distal level comprised demographic and socioeconomic variables. Variables associated with prison conditions were included in the second level. In the third level, the most proximal, were included variables related to the history of mental health and life habits. Variables were selected for the final model using the backward Euler method. Following this method, the variables were placed in the model according to their hierarchical level, and those that had a value $\mathrm{p} \leq 0.20$ were maintained.

This study was authorized by the $5^{\text {th }}$ RPS and approved by the Research Ethics Committee in the Health Area (Cepas) of the Federal University of Rio Grande (FURG) under the $n^{\circ} 05 / 2017$. The participation was voluntary and happened following accordance to the Informed Consent Form (ICF).

\section{Results}

Among the 643 people interviewed for this research, 90.2\% were male, with a mean age of 33.2 years ( $S D \pm 9.2$ years), $62.3 \%$ had no partner, $60.9 \%$ were white and $57.5 \%$ had a religion, being Catholicism the most prevalent one (48\%). The income of $53.9 \%$ of the sample was of at least 1.1 minimum wages and the average study-years were 6.3 years ( $\mathrm{SD} \pm 2.8$ years). The average length of the sentence, including all convictions, was of 73 months $(\mathrm{SD} \pm 69$ months), $66.8 \%$ were recidivist prisoners and $61.3 \%$ did not work in prison (Table 1).

Regarding mental health, $9.6 \%$ of the interviewees reported having a history of mental illness and $18.2 \%$ reported having a family member with history of mental illness. Smokers accounted for $60.3 \%$ of the sample and the average number of cigarettes per day was of 17.4 cigarettes ( $\mathrm{SD} \pm 12.5$ cigarettes). Concerning alcohol consumption, $56.2 \%$ of the sample stated that they did not consume alcohol before arrest, and among those who consumed it, the average frequency was of 2.9 days per week ( $\mathrm{SD} \pm 2.1$ days). Drug usage was reported by $76.2 \%$ of the inmates, being marijuana $(80.2 \%)$, cocaine $(62.8 \%)$ and crack (44\%) the most prevalent ones (Table 1).

The prevalence of depression among studied prisoners was of 20.6\% (CI 95\%: 17.5-23.8) (Table 2). After adjusted analysis it was observed that being a woman (RP: 2.14; 95\% CI: 1.52-3.01) and having a history of mental illness (RP: 2.15; CI 95\%: 1.56-2.95) increased probability of depression by 114 and $115 \%$, respectively. Having black or brown skin color (PR: 1.37; CI 95\% 1.02-1.86) increased the probability of depression by $37 \%$ over those of white skin color. Not having a religion (PR: 0.67; CI 95\%: 0.49-0.92) decreased the probability of having depression by $33 \%$, while not receiving visits (PR: 1.36; CI 95\%: 1.00-1.86) increased the probability by $36 \%$. Drug use (PR: 1.77; CI 95\%: 1.14-2.75) and smoking (PR: 1.89; CI 95\%: $1.30-2.76)$ increased the probability of having depression in $77 \%$ and $89 \%$, respectively. In addition, not practicing physical activities in prison (PR: 1.44; CI95\%: 1.06-1.96) also increased the probability of having depression by $44 \%$ (Table 3 ).

Generalized anxiety was reported by $19.9 \%$ (CI 95\%: 16.8-23.0) of the interviewees (Table 2). After adjusted analysis it was observed that being female (RP 2.34; CI 95\%: 1.66-3.30) increased the probability of having anxiety in $134 \%$. History of mental illness (RP: 1.62 ; CI 95\%: 1.09-2.40) or family history of mental illness (RP: 1.50; CI 95\%: $1.07-2.11$ ) increased the probability of having anxiety by 62 and 50\%, respectively. Smoking (RP: 1.59; CI 95\%: 1.10-2.28) and drug use (RP: 1.54; CI 95\%: 1.02-2.34) also increased the probability of having anxiety in 59 and 54\%, respectively (Table 4).

\section{Discussion}

The prevalence of depression found in this study was of $20.6 \%$ among 643 interviewees, using the MINI plus questionnaire ${ }^{25}$. In international literature, rates of depression among prisoners range from 5 to $72.6 \%{ }^{13,16}$. It is believed that these differences can be explained due to different methodologies and cultural aspects of the 
Table 1. Population description of the prevalence study and factors associated with depression and anxiety in prisoners in southern Brazil. $2018(n=643)$

\begin{tabular}{|c|c|c|}
\hline Variables & $\mathrm{N}$ & $\%$ \\
\hline $\begin{array}{l}\text { Sex } \\
\text { Male } \\
\text { Female }\end{array}$ & $\begin{array}{c}580 \\
63\end{array}$ & $\begin{array}{c}90.2 \\
9.8\end{array}$ \\
\hline $\begin{array}{l}\text { Age (years) } \\
\leq 25 \\
26-35 \\
\geq 36\end{array}$ & $\begin{array}{l}140 \\
281 \\
221\end{array}$ & $\begin{array}{l}21.8 \\
43.8 \\
34.4\end{array}$ \\
\hline $\begin{array}{l}\text { Skin Color } \\
\text { White } \\
\text { Not White }\end{array}$ & $\begin{array}{l}380 \\
244 \\
\end{array}$ & $\begin{array}{l}60.9 \\
39.1 \\
\end{array}$ \\
\hline $\begin{array}{l}\text { Marital status } \\
\text { Single } \\
\text { Married } \\
\end{array}$ & $\begin{array}{l}400 \\
242 \\
\end{array}$ & $\begin{array}{l}37.7 \\
62.3 \\
\end{array}$ \\
\hline $\begin{array}{l}\text { Remuneration (before arrested) } \\
\geq 1.1 \text { minimum wage } \\
\leq 1 \text { minimum wage }\end{array}$ & $\begin{array}{l}342 \\
292 \\
\end{array}$ & $\begin{array}{l}53.9 \\
46.1 \\
\end{array}$ \\
\hline $\begin{array}{l}\text { Study (in years) } \\
\geq 9 \\
6-8 \\
\leq 5\end{array}$ & $\begin{array}{l}112 \\
264 \\
260\end{array}$ & $\begin{array}{l}17.6 \\
41.5 \\
40.9\end{array}$ \\
\hline $\begin{array}{l}\text { Work in prison } \\
\text { Yes } \\
\text { No }\end{array}$ & $\begin{array}{l}247 \\
392\end{array}$ & $\begin{array}{l}38.7 \\
61.3\end{array}$ \\
\hline $\begin{array}{l}\text { Crime } \\
\text { Homicide } \\
\text { Robbery } \\
\text { Drug } \\
\text { Traffic } \\
\text { Others* }\end{array}$ & $\begin{array}{l}97 \\
159 \\
217 \\
163\end{array}$ & $\begin{array}{l}15.3 \\
25.0 \\
34.1 \\
25.6\end{array}$ \\
\hline $\begin{array}{l}\text { Time in prison (in months) } \\
\geq 61 \\
31-60 \\
\leq 30\end{array}$ & $\begin{array}{l}263 \\
140 \\
218\end{array}$ & $\begin{array}{l}42.4 \\
22.5 \\
35.1\end{array}$ \\
\hline $\begin{array}{l}\text { Receives visits } \\
\text { Yes } \\
\text { No }\end{array}$ & $\begin{array}{l}447 \\
194 \\
\end{array}$ & $\begin{array}{l}69.7 \\
30.3 \\
\end{array}$ \\
\hline $\begin{array}{l}\text { Religion } \\
\text { No } \\
\text { Yes }\end{array}$ & $\begin{array}{l}368 \\
272 \\
\end{array}$ & $\begin{array}{l}57.5 \\
42.5 \\
\end{array}$ \\
\hline $\begin{array}{l}\text { History of mental illness } \\
\text { No } \\
\text { Yes }\end{array}$ & $\begin{array}{c}577 \\
61 \\
\end{array}$ & $\begin{array}{c}90.4 \\
9.6\end{array}$ \\
\hline $\begin{array}{l}\text { History of mental illness in the Family } \\
\text { No } \\
\text { Yes }\end{array}$ & $\begin{array}{l}520 \\
116\end{array}$ & $\begin{array}{l}81.8 \\
18.2\end{array}$ \\
\hline $\begin{array}{l}\text { Smoker } \\
\text { No } \\
\text { Yes }\end{array}$ & $\begin{array}{l}254 \\
386\end{array}$ & $\begin{array}{l}39.7 \\
60.3\end{array}$ \\
\hline $\begin{array}{l}\text { Alcohol use (in a lifetime) } \\
\text { No } \\
\text { Yes }\end{array}$ & $\begin{array}{l}358 \\
279\end{array}$ & $\begin{array}{l}56.2 \\
43.8\end{array}$ \\
\hline $\begin{array}{l}\text { Illicit drug use (in a lifetime) } \\
\text { No } \\
\text { Yes }\end{array}$ & $\begin{array}{l}151 \\
490\end{array}$ & $\begin{array}{l}23.6 \\
76.4\end{array}$ \\
\hline $\begin{array}{l}\text { Physical Activities } \\
\text { Yes } \\
\text { No }\end{array}$ & $\begin{array}{l}386 \\
254\end{array}$ & $\begin{array}{l}39.7 \\
60.3\end{array}$ \\
\hline
\end{tabular}

Subtitles: ${ }^{*}$ Sexual crimes, gun possession, receiving, rustling and bodily injury.
Table 3. Crude and adjusted analyses of factors associated with depression in prison population in southern Brazil, 2018 ( $n=643$ )

\begin{tabular}{|c|c|c|c|}
\hline Variables & $\%$ & Crude PR (95\% CI) & $\begin{array}{c}\text { Adjusted PR } \\
(95 \% \mathrm{CI})\end{array}$ \\
\hline $\begin{array}{l}\text { Sex } \\
\text { Male } \\
\text { Female }\end{array}$ & $\begin{array}{l}18.4 \\
41.3\end{array}$ & $\begin{array}{c}p<0.001 \\
1.00 \\
2.24(1.59-3.15)\end{array}$ & $\begin{array}{c}\mathrm{P}<0.001 \\
1.00 \\
2.14(1.52-3.01)\end{array}$ \\
\hline $\begin{array}{l}\text { Age (years) } \\
\leq 25 \\
26-35 \\
\geq 36\end{array}$ & $\begin{array}{l}19.3 \\
22.4 \\
19.5\end{array}$ & $\begin{array}{c}p=0.643 \\
1.00 \\
1.16(0.78-1.74) \\
1.01(0.65-1.55)\end{array}$ & $\begin{array}{c}p=0.511 \\
1.00 \\
1.18(0.78-1.79) \\
0.98(0.61-1.57)\end{array}$ \\
\hline \begin{tabular}{|l} 
Skin Color \\
White \\
Not White \\
\end{tabular} & $\begin{array}{l}17.9 \\
25.4 \\
\end{array}$ & $\begin{array}{c}p=0.024 \\
1.00 \\
1.42(1.05-1.93)\end{array}$ & $\begin{array}{c}p=0.036 \\
1.00 \\
1.37(1.02-1.86)\end{array}$ \\
\hline $\begin{array}{l}\text { Marital status } \\
\text { Single } \\
\text { Married }\end{array}$ & $\begin{array}{l}21.8 \\
19.0 \\
\end{array}$ & $\begin{array}{c}p=0.406 \\
1.00 \\
1.14(0.83-1.58)\end{array}$ & $\begin{array}{c}p=0.307 \\
1.00 \\
1.18(0.85-1.64)\end{array}$ \\
\hline $\begin{array}{l}\text { Remuneration (before arrested) } \\
\geq 1.1 \text { minimum wage } \\
\leq 1 \text { minimum wage } \\
\end{array}$ & $\begin{array}{l}22.8 \\
18.8 \\
\end{array}$ & $\begin{array}{c}p=0.221 \\
1.00 \\
0.83(0.61-1.12)\end{array}$ & $\begin{array}{c}p=0.100 \\
1.00 \\
0.77(0.56-1.05)\end{array}$ \\
\hline $\begin{array}{l}\text { Study (in years) } \\
\geq 9 \\
6-8 \\
\leq 5\end{array}$ & $\begin{array}{l}21.4 \\
23.1 \\
18.1\end{array}$ & $\begin{array}{c}p=0.358 \\
1.00 \\
1.08(0.71-1.64) \\
0.84(0.54-1.31)\end{array}$ & $\begin{array}{c}p=0.257 \\
1.00 \\
1.19(0.79-1.80) \\
0.90(0.59-1.39)\end{array}$ \\
\hline $\begin{array}{l}\text { Work in prison } \\
\text { Yes } \\
\text { No }\end{array}$ & $\begin{array}{l}22.7 \\
17.8 \\
\end{array}$ & $\begin{array}{c}p=0.138 \\
1.00 \\
1.27(0.92-1.76)\end{array}$ & $\begin{array}{c}p=0.215 \\
1.00 \\
1.22(0.88-1.69)\end{array}$ \\
\hline $\begin{array}{l}\text { Crime } \\
\text { Homicide } \\
\text { Robbery } \\
\text { Drug } \\
\text { Traffic } \\
\text { Others* } \\
\end{array}$ & $\begin{array}{l}13.4 \\
20.8 \\
24.0 \\
20.9\end{array}$ & $\begin{array}{c}p=0.208 \\
1.00 \\
1.55(0.86-2.79) \\
1.79(1.02-3.13) \\
1.56(0.86-2.80)\end{array}$ & $\begin{array}{c}p=0.312 \\
1.00 \\
1.70(0.92-3.13) \\
1.70(0.95-3.03) \\
1.54(0.84-2.82)\end{array}$ \\
\hline $\begin{array}{l}\text { Time in prison (in months) } \\
\geq 61 \\
31-60 \\
\leq 30\end{array}$ & $\begin{array}{l}18.6 \\
22.9 \\
21.6\end{array}$ & $\begin{array}{c}p=0.554 \\
1.00 \\
1.23(0.83-1.82) \\
1.16(0.81-1.65)\end{array}$ & $\begin{array}{c}p=0.553 \\
1.0 \\
1.24(0.83-1.84) \\
1.08(0.75-1.55)\end{array}$ \\
\hline $\begin{array}{l}\text { Receives visits } \\
\text { Yes } \\
\text { No }\end{array}$ & $\begin{array}{l}18.1 \\
26.8\end{array}$ & $\begin{array}{c}p=0.013 \\
1.00 \\
1.48(1.09-2.01)\end{array}$ & $\begin{array}{c}p=0.048 \\
1.00 \\
1.36(1.00-1.86)\end{array}$ \\
\hline $\begin{array}{l}\text { Religion } \\
\text { No } \\
\text { Yes }\end{array}$ & $\begin{array}{l}16.2 \\
24.2 \\
\end{array}$ & $\begin{array}{c}p=0.014 \\
1.00 \\
1.50(1.08-2.07)\end{array}$ & $\begin{array}{c}p=0.013 \\
1.00 \\
1.63(1.07-2.48)\end{array}$ \\
\hline $\begin{array}{l}\text { History of mental illness } \\
\text { No } \\
\text { Yes }\end{array}$ & $\begin{array}{l}17.5 \\
50.8\end{array}$ & $\begin{array}{c}p<0.001 \\
1.00 \\
2.90(2.14-3.93)\end{array}$ & $\begin{array}{c}p=<0.001 \\
1.00 \\
2.15(1.56-2.95)\end{array}$ \\
\hline $\begin{array}{l}\text { History of mental illness in } \\
\text { the Family } \\
\text { No } \\
\text { Yes }\end{array}$ & $\begin{array}{l}18.5 \\
31.0 \\
\end{array}$ & $\begin{array}{c}p=0.002 \\
1.00 \\
1.68(1.21-2.33)\end{array}$ & $\begin{array}{c}p=0.087 \\
1.00 \\
1.33(0.95-1.85)\end{array}$ \\
\hline $\begin{array}{l}\text { Smoker } \\
\text { No } \\
\text { Yes }\end{array}$ & $\begin{array}{l}11.4 \\
26.9\end{array}$ & $\begin{array}{c}p<0.001 \\
1.00 \\
2.36(1.61-3.45)\end{array}$ & $\begin{array}{c}p=0.001 \\
1.00 \\
1.89(1.30-2.76)\end{array}$ \\
\hline $\begin{array}{l}\text { Alcohol use (in a lifetime) } \\
\text { No } \\
\text { Yes } \\
\end{array}$ & $\begin{array}{l}19.8 \\
21.5\end{array}$ & $\begin{array}{c}p=0.604 \\
1.00 \\
1.08(0.80-1.47) \\
\end{array}$ & $\begin{array}{c}p=0.541 \\
1.00 \\
0.91(0.68-1.22)\end{array}$ \\
\hline $\begin{array}{l}\text { Illicit drug use (in a lifetime) } \\
\text { No } \\
\text { Yes }\end{array}$ & $\begin{array}{l}13.9 \\
22.9 \\
\end{array}$ & $\begin{array}{c}p=0.018 \\
1.00 \\
1.64(1.07-2.52)\end{array}$ & $\begin{array}{c}p=0.011 \\
1.00 \\
1.77(1.14-2.75)\end{array}$ \\
\hline $\begin{array}{l}\text { Physical Activities } \\
\text { Yes } \\
\text { No }\end{array}$ & $\begin{array}{l}16.3 \\
27.6 \\
\end{array}$ & $\begin{array}{c}p<0.001 \\
1.00 \\
1.69(1.25-2.28) \\
\end{array}$ & $\begin{array}{c}p=0.018 \\
1.00 \\
1.44(1.06-1.96)\end{array}$ \\
\hline
\end{tabular}

Subtitles: * Sexual crimes, gun possession, receiving, rustling and bodily injury.

Table 2. Prevalence of depression and anxiety in prisoners in southern Brazil, 2018 ( $n=643$ )

\begin{tabular}{|l|c|c|}
\hline Mental Disorders & $\%$ & Cl 95\% \\
\hline Depression & 20.6 & $17.5-23.8$ \\
\hline Anxiety & 19.9 & $16.8-23.0$ \\
\hline
\end{tabular}


Table 4. Crude and adjusted analyses of factors associated with anxiety in prison population in southern Brazil, 2018 ( $n=643)$

\begin{tabular}{|c|c|c|c|}
\hline Variables & $\%$ & Crude PR (95\% CI) & $\begin{array}{c}\text { Adjusted PR } \\
(95 \% \mathrm{Cl})\end{array}$ \\
\hline $\begin{array}{l}\text { Sex } \\
\text { Male } \\
\text { Female }\end{array}$ & $\begin{array}{l}17.6 \\
41.3 \\
\end{array}$ & $\begin{array}{c}p<0.001 \\
1.00 \\
2.35(1.66-3.31) \\
\end{array}$ & $\begin{array}{c}p=<0.001 \\
1.00 \\
2.34(1.66-3.30)\end{array}$ \\
\hline $\begin{array}{l}\text { Age (years) } \\
\leq 25 \\
26-35 \\
\geq 36\end{array}$ & $\begin{array}{l}18.6 \\
21.7 \\
18.6 \\
\end{array}$ & $\begin{array}{c}p=0.612 \\
1.00 \\
1.17(0.77-1.76) \\
1.00(0.64-1.56)\end{array}$ & $\begin{array}{c}p=0.371 \\
1.00 \\
1.13(0.75-1.71) \\
0.88(0.56-1.37)\end{array}$ \\
\hline $\begin{array}{l}\text { Skin Color } \\
\text { White } \\
\text { Not White }\end{array}$ & $\begin{array}{l}18.9 \\
21.7\end{array}$ & $\begin{array}{c}p=0.398 \\
1.00 \\
1.15(0.84-1.57)\end{array}$ & $\begin{array}{c}p=0.637 \\
1.00 \\
1.07(0.78-1.48)\end{array}$ \\
\hline $\begin{array}{l}\text { Marital status } \\
\text { Single } \\
\text { Married }\end{array}$ & $\begin{array}{l}18.0 \\
23.1\end{array}$ & $\begin{array}{c}p=0.114 \\
1.00 \\
1.29(0.94-1.75)\end{array}$ & $\begin{array}{c}p=0.233 \\
1.00 \\
0.82(0.60-1.12)\end{array}$ \\
\hline $\begin{array}{l}\text { Remuneration (before arrested) } \\
\geq 1.1 \text { minimum wage } \\
\leq 1 \text { minimum wage }\end{array}$ & $\begin{array}{l}20.8 \\
19.5\end{array}$ & \begin{tabular}{c|}
$p=0.698$ \\
1.00 \\
$1.06(0.78-1.45)$ \\
\end{tabular} & $\begin{array}{c}p=0.345 \\
1.00 \\
0.85(0.62-1.17)\end{array}$ \\
\hline $\begin{array}{l}\text { Study (in years) } \\
\geq 9 \\
6-8 \\
\leq 5\end{array}$ & $\begin{array}{l}20.5 \\
23.1 \\
16.5\end{array}$ & $\begin{array}{c}p=0.168 \\
1.00 \\
1.40(0.98-1.98) \\
0.81(0.51-1.27)\end{array}$ & $\begin{array}{c}\mathrm{p}=0.724 \\
1.00 \\
1.26(0.82-1.94) \\
0.92(0.57-1.46)\end{array}$ \\
\hline $\begin{array}{l}\text { Work in prison } \\
\text { Yes } \\
\text { No }\end{array}$ & $\begin{array}{l}18.6 \\
20.7\end{array}$ & $\begin{array}{c}p=0.529 \\
1.00 \\
1.11(0.80-1.54) \\
\end{array}$ & $\begin{array}{c}p=0.451 \\
1.00 \\
1.13(0.81-1.56)\end{array}$ \\
\hline $\begin{array}{l}\text { Crime } \\
\text { Homicide } \\
\text { Robbery } \\
\text { Drug } \\
\text { Traffic } \\
\text { Others* }\end{array}$ & $\begin{array}{l}16.5 \\
21.4 \\
20.7 \\
19.6\end{array}$ & $\begin{array}{c}p=0.796 \\
1.00 \\
1.30(0.76-2.22) \\
1.26(0.75-2.11) \\
1.19(0.69-2.05)\end{array}$ & $\begin{array}{c}\mathrm{p}=0.574 \\
1.00 \\
1.39(0.81-2.41) \\
1.09(0.65-1.84) \\
1.17(0.68-2.04)\end{array}$ \\
\hline $\begin{array}{l}\text { Time in prison (in months) } \\
\geq 61 \\
31-60 \\
\leq 30\end{array}$ & $\begin{array}{l}19.4 \\
20.7 \\
20.6 \\
\end{array}$ & $\begin{array}{c}\mathrm{p}=0.926 \\
1.00 \\
1.07(0.71-1.60) \\
1.06(0.74-1.52) \\
\end{array}$ & $\begin{array}{c}p=0.917 \\
1.00 \\
1.09(0.72-1.64) \\
1.02(0.71-1.46)\end{array}$ \\
\hline $\begin{array}{l}\text { Receives visits } \\
\text { Yes } \\
\text { No }\end{array}$ & $\begin{array}{l}19.0 \\
22.2\end{array}$ & \begin{tabular}{c|}
$p=0.352$ \\
1.00 \\
$1.17(0.84-1.62)$ \\
\end{tabular} & $\begin{array}{c}p=0.942 \\
1.00 \\
1.01(0.72-1.42)\end{array}$ \\
\hline $\begin{array}{l}\text { Religion } \\
\text { No } \\
\text { Yes }\end{array}$ & $\begin{array}{l}19.5 \\
20.4 \\
\end{array}$ & $\begin{array}{c}p=0.780 \\
1.00 \\
1.05(0.76-1.43) \\
\end{array}$ & $\begin{array}{c}p=0.741 \\
1.00 \\
0.99(0.65-1.49)\end{array}$ \\
\hline $\begin{array}{l}\text { History of mental illness } \\
\text { No } \\
\text { Yes }\end{array}$ & $\begin{array}{l}17.9 \\
39.3 \\
\end{array}$ & $\begin{array}{c}p<0.001 \\
1.00 \\
1.77(1.28-2.46) \\
\end{array}$ & $\begin{array}{c}p=0.016 \\
1.00 \\
1.62(1.09-2.40)\end{array}$ \\
\hline $\begin{array}{l}\text { History of mental illness in } \\
\text { the Family } \\
\text { No } \\
\text { Yes }\end{array}$ & $\begin{array}{l}17.5 \\
31.0 \\
\end{array}$ & $\begin{array}{c}p<0.001 \\
1.00 \\
2.20(1.54-3.15) \\
\end{array}$ & $\begin{array}{c}p=0.016 \\
1.00 \\
1.50(1.07-2.11)\end{array}$ \\
\hline $\begin{array}{l}\text { Smoker } \\
\text { No } \\
\text { Yes }\end{array}$ & $\begin{array}{l}13.4 \\
24.4 \\
\end{array}$ & \begin{tabular}{c|}
$\mathrm{p}<0.001$ \\
1.00 \\
$1.82(1.27-2.60)$ \\
\end{tabular} & $\begin{array}{c}p=0.012 \\
1.00 \\
1.59(1.10-2.28)\end{array}$ \\
\hline $\begin{array}{l}\text { Alcohol use (in a lifetime) } \\
\text { No } \\
\text { Yes }\end{array}$ & $\begin{array}{l}17.9 \\
22.6 \\
\end{array}$ & $\begin{array}{c}p=0.140 \\
1.00 \\
1.26(0.93-1.72) \\
\end{array}$ & $\begin{array}{c}p=0.452 \\
1.00 \\
1.12(0.82-1.52)\end{array}$ \\
\hline $\begin{array}{l}\text { Illicit drug use (in a lifetime) } \\
\text { No } \\
\text { Yes }\end{array}$ & $\begin{array}{l}15.2 \\
21.4\end{array}$ & \begin{tabular}{c|}
$\mathrm{p}=0.096$ \\
1.00 \\
$1.41(0.93-2.13)$ \\
\end{tabular} & $\begin{array}{c}p=0.039 \\
1.00 \\
1.54(1.02-2.34)\end{array}$ \\
\hline $\begin{array}{l}\text { Physical Activities } \\
\text { Yes } \\
\text { No }\end{array}$ & $\begin{array}{l}16.8 \\
24.9\end{array}$ & $\begin{array}{c}p=0.014 \\
1.0 \\
1.47(1.08-2.00)\end{array}$ & $\begin{array}{c}p=0.165 \\
1.00 \\
1.24(0.91-1.70)\end{array}$ \\
\hline
\end{tabular}

Subtitles: * Sexual crimes, gun possession, receiving, rustling and bodily injury. population. However, a survey with 1,809 prisoners in the city of São Paulo using the same methodology of this study reported a prevalence of $14.7 \%$ for depression ${ }^{4}$. A study with 497 men in Salvador, also using the MINI questionnaire, found a rate of depression of $17.6 \%{ }^{26}$. In Santa Catarina, when interviewing 557 inmates with same questionnaire, the reported prevalence of depression was of $16 \%{ }^{27}$. It is observed that when the same instrument for mental disorder evaluation is used, the proportion of disease detection presents variations probably related to transculturality and not methodology. In addition, it is pointed out that the prevalence found in this study confirms that depression is more frequent among the population deprived of liberty, being almost four times higher than in the general Brazilian population, which is of $5.8 \%{ }^{8}$. This fact can be justified by greater exposure of this population to risk factors such as low socioeconomic status, unemployment, substance abuse and violence ${ }^{6}$.

Another disorder investigated in this study was generalized anxiety, which had a predominance of $19.9 \%$. As observed in depression, prevalence of anxiety among the population deprived of liberty also varies widely among studies, from 6.9 to $77.8 \%$. The use of different methodologies and instruments make it difficult to compare findings. Brazilian studies that used the same methodology as ours reported anxiety rates that ranged from 6.9 to $27.7 \%$. One of the reasons for this variation might be because these studies were performed in different regions of the country. For example, in major urban centers the organized crime commands prisons and conflicts are frequent, which differs from what is observed in smaller cities.

This study also corroborates that anxiety among prisoners is more prevalent than in the general population, reaching twice the ratio of the Brazilian citizens, which is of $9.3 \%{ }^{8}$. The more widespread anxiety among prisoners can be a result of increased exposure to factors such as deprivation of social interaction, deprivation of liberty, rigid rules and the constant control of the individuals, changes of environment and stressful situations ${ }^{28}$.

Being a woman was identified as a major risk factor for depression and anxiety, doubling the probability of suffering from these diseases when compared to men. This phenomenon is also observed in the general population ${ }^{8}$, which probably is reflected in female population deprived of liberty. In one of the mentioned studies ${ }^{27}$, where 466 men and 91 women were interviewed, twice as many mental disorders were found in women, and the prevalence of depression reported was of $13.7 \%$ in men and $27.5 \%$ in women. Another study that included with 1110 men and 463 women in Rio de Janeiro found that the depression rate among women was $16 \%$ higher than among men ${ }^{17}$. There might be several reasons for this, such as constant hormonal influence in women, more exposure to domestic and sexual violence and especially the societal role assigned to women as caregiver.

Another important factor for the occurrence of the depression and anxiety was having a personal history of mental illness, increasing probability of having depression by $115 \%$ and anxiety by $62 \%$. A study carried out in Rio Grande do Sul, Brazil, with 287 women, found that the odds of suffering from depression was three times higher among inmates with personal history of mental illness ${ }^{6}$. Another study conducted in Egypt with 1,350 prisoners also reported that inmates with a history of mental illness showed twice the probability of developing some psychiatric disorder ${ }^{19}$. There are few studies in literature that associate depression and anxiety with having a history of mental illness, possibly due to the difficulty of accessing medical records from before arrest. In our study we used the question "Have you ever had any mental illness?" to collect this data. It is believed that it is important to collect this information when assessing the presence current disorders, as there is a possibility that some prisoners may have previous mental illnesses worsened due to a lack of adequate diagnosis and treatment. Having previous mental illnesses can increase the probability of having depression during incarceration for various reasons, including interruption of treatment. Other factors associated with prison life can also be identified as triggers, such as withdrawal from family life, limited access to leisure activities, exposure to an unhealthy environment and difficulty to receive appropriate treatment. 
In addition to being a woman and having a history of mental illness, smoking and drug use were also risk factors for depression, increasing the odds by $89 \%$ and $77 \%$, respectively, and for anxiety, by $59 \%$ and $49 \%$. Another study in India comparing 122 psychiatric prisoners with 122 non-psychiatric prisoners also confirmed a $21 \%$ increase in smoking among those who had some mental illness 29. Concerning anxiety, a survey of 649 prisoners in Ethiopia found that smoking increased 2.6 times the probability of having the disorder ${ }^{22}$. In another study conducted with 60 prisoners in Rio Grande do $\mathrm{Sul}^{30}$, a strong correlation was found between depressive symptoms and drug use. The study also states that the higher the drug use, the higher the irritation, aggressiveness, level of depressive symptoms and recurrence of crimes, and vice versa. However, in both cases it is not possible to state whether this is a relationship of cause or effect. We believe that depressed and anxious people are at greater risk of consuming cigarettes and drugs as an attempt to reduce their symptoms, entering in a vicious cycle.

Factors that presented an increased risk only for depression were having brown/black skin color, not receiving visits and not practicing physical activity. Supporting these results, a systematic review that included 14 Brazilian studies showed a higher prevalence of mental disorders in non-white people. The study also concludes that there is no biological relationship between race and mental health. However, it is necessary to investigate the populations that are more likely to suffer from mental diseases, aiming to investigate risk factors related to environment or social context in order to prevent them ${ }^{31}$. In this research, the majority of prisoners were white-skinned, which is consistent with the profile of prisoners in southern Brazil, but contrasts with the profile of prison populations in rest of the country, where majority of the inmates have brown and black skin colors. We did not identify any study in the literature that associated skin color with depression in the population deprived of liberty. We believe that perhaps because the non-white population is a minority, it may be more exposed to discrimination, marginalization and consequent social vulnerability both outside and inside prison, which could increase the probability of having depression. Not receiving visits was also identified as a risk factor for depression, and this is widely discussed in literature. Studies in Mexico and Egypt state that receiving visits reduces the risk of having some mental disorder in $37 \%$ and $50 \%$, respectively ${ }^{14,19}$. Frequent visits represent strong support network and family bond, both fundamental elements for the promotion of mental health. Another risk factor for depression was not practicing physical activity. A case-control study with 64 Italian prisoners subjected to a nine-month exercise program showed that physical activity significantly reduced the prevalence of depression when compared to the control group, among which the disease even became more prevalent ${ }^{32}$. Physical activity is an important ally in the prevention and promotion of mental health, considering its potential in regulating mood and promoting physical and psychological well-being.

Having family history of mental illness increased the probability of having anxiety by $50 \%$. We did not identify in the literature any study that directly related these factors, however a study carried out in Brazil with female prisoners associated the occurrence of depression both with individual and familiar history of mental illness ${ }^{6}$. One hypothesis is that it occurs because the inmates have limited contact with family members, and they are unable to financially or emotionally contribute with family care. It is known that having parents with a history of mental illness during childhood can increase the probability of developing mental disorders in adulthood ${ }^{33}$. In addition to the genetic factor, living from early age with a relative that suffers from a severe mental disorder can have influence in the development, personality and family structure of the individuals, also increasing their exposure to risk factors such as violence and negligence.

As discussed previously, there are some limitations in the acquisition of these data. However, it is important to seek them whenever possible, in order to direct actions of prevention and promotion of mental health focused on those identified with this risk factor
Not having a religion was a protective factor in this study for depression, decreasing the probability of reporting it by $33 \%$. However, literature points to religion as a protective factor for mental illness ${ }^{17,34}$. A study of 1,573 prisoners in Rio de Janeiro showed that having no religion increased the risk of depression in $79 \%{ }^{17}$. Another study with 213 women in United States of America showed that inmates participating in religious groups had lower depression scale scores than those who were not religious ${ }^{34}$. In our study, the prevalence of prisoners who reported having a religion was of $57.2 \%$, which is considerably lower in comparison to the rates found in literature, that varied from 70.3 to $79.6 \% 1733$. In addition, we may have faced a reverse causality effect, where depressed prisoners pursued a religion more than those without the disease.

Based on the obtained results, the implementation of preventive actions for depression and anxiety are suggested, especially among prisoners that are women and that have a history of mental illness, considering that these risk factors doubled the probability of suffering from these disorders. We suggest that the patients with history of mental illnesses are identified when imprisoned, being monitored by the local health professionals. Prisoners without history of mental illnesses that start showing symptoms for these complications should also be evaluated and receive proper treatment.

It is believed that the need to implement strategies to strengthen family bonds and support networks are essential to avoid or reduce mental illness during imprisonment and during the process of resocialization. Another suggestion is to offer actions that contribute against the use of tobacco and other drugs, such as the National Program of Tobacco Control, that is already offered by the Basic Health Units of some of the researched cities, as well as the rehabilitation treatment for chemical dependency that is offered in partnership with services such as the Centers of Psychosocial Attention (CAPS). However, one of the main challenges faced during the study was the lack of structure of the prisons. Due to overcrowding, places that were originally intended to be used as classrooms or libraries were assigned to other uses, which hinders the realization of group activities. This is the main challenge for the realization of actions aiming to promote health and prevent illnesses inside the prisons included in our research.

It is also worth noting the relevance of the present study, as it was carried out in six different cities, in a region where there are still no published data on this topic and for using the MINI-Plus, an individual clinical interview instrument based on the DSM V criteria for diagnostics. Even though it is one of the most complete instruments, MINI-Plus is seldom used in prison context due to logistical difficulties. However, it is necessary to consider some limitations. Because it presents a cross-sectional design, it prevents the establishment relationships of cause and effect. Some examples are the variables for religion and drug use. The first case leads us to hypothesize a case of reverse causality, where depressed people would be more likely to seek support in religion. In the second case, it is not possible to say whether drug use increases the prevalence of anxiety and depression or if it is a symptom of the disorder. Despite the limitations, the results suggest the viability of the adopted methodology, since other studies on mental disorders have used this same method.

The present study confirmed the high prevalence of mental disorders in population deprived of liberty and pointed out several risk factors that can help in planning effective strategies for prevention and promotion of mental health. Other studies are needed to explore this topic, given its complexity and scarcity of scientific knowledge on the subject.

\section{References}

1. Walmsley R. World Population List. 11th. London: Institute for Criminal Justice Research; 2016.

2. Brasil. Ministério da Justiça. Departamento Penitenciário. Levantamento Nacional de Informações Peniténciárias atualizado. Brasília - DF; 2016.

3. Marin-Basallote N, Navarro-Repiso C. Study of the prevalence of severe mental disorder in the penitentiaries Puerto I, II and III of Puerto de 
Santa Maria (Cadiz): new strategies of psychiatric care in prison. Rev Esp Sanid Penit. 2012;14:80-5.

4. Andreoli SB, Dos Santos MM, Quintana MI, Ribeiro WS, Blay SL, Taborda JG, et al. Prevalence of mental disorders among prisoners in the state of Sao Paulo, Brazil. PloS One. 2014;9:e88836.

5. Zabala-Banos MC, Segura A, Maestre-Miquel C, Martinez-Lorca M, Rodriguez- Martin B, Romero D, et al. Mental disorder prevalence and associated risk factors in three prisons of Spain. Rev Esp Sanid Penit. 2016;18:13-23.

6. Canazaro D, Lima A, II. Characteristics, depressive symptoms, and associated factors in incarcerated women in the State of Rio Grande do Sul, Brazil. Cad Saude Publica. 2010;26:1323-33.

7. American Psychiatric Association. DSM-5: manual diagnóstico e estatístico de transtornos mentais. 5a ed. Porto Alegre: Artmed; 2014.

8. World Health Organization. Depression and Other Common Mental Disorders Global Health Estimates. Geneva: World Health Organization; 2017.

9. Instituto Brasileiro de Geografia e Estatística. Pesquisa Nacional de Saúde 2013. Rio de Janeiro: IBGE; 2013.

10. Cunha RV, Bastos, GA N, Duca GF Prevalência de depressão e fatores associados em comunidade de baixa renda de Porto Alegre, Rio Grande do Sul. Rev Bras Epidemiol. 2012;15(2):346-54.

11. Uribe Rodríguez AF, Martínez Rodríguez JM. Depresión y ansiedad estado/rasgo en internos adscritos al "Programa de Inducción al Tratamiento Penitenciario" en Bucaramanga, Colombia. Rev Criminalidad. 2012;54:47-60.

12. Unver Y, Yuce M, Bayram N, Bilgel N. Prevalence of depression, anxiety, stress, and anger in Turkish prisoners. J Forensic Sci. 2013;58:1210-8.

13. Osasona SO, Koleoso ON. Prevalence and correlates of depression and anxiety disorder in a sample of inmates in a Nigerian prison. Int J Psychiatry Med. 2015;50:203-18.

14. Albertie A, Bourey C, Stephenson R, Bautista-Arredondo S. Connectivity, prison environment and mental health among first-time male inmates in Mexico City. Global Public Health. 2017;12:170-84.

15. Reed E, Raj A, Falbo G, Caminha F, Decker MR, Kaliel DC, et al. The prevalence of violence and relation to depression and illicit drug use among incarcerated women in Recife, Brazil. Int J Law Psychiatry. 2009;32:323-8.

16. Mir J, Kastner S, Priebe S, Konrad N, Strohle A, Mundt AP. Treating substance abuse is not enough: comorbidities in consecutively admitted female prisoners. Addict Behav. 2015;46:25-30.

17. Constantino P, Assis SG, Pinto LW. The impact of prisons on the mental health of prisoners in the state of Rio de Janeiro, Brazil. Ciênc Saude Coletiva. 2016;21:2089-100.

18. Pulido-Criollo F, Rodriguez-Landa JF, Colorado-Martinez MP. Sociodemographic factors associated with symptoms of depression in a sample women held at two prisons in Mexico. Rev Panam Salud Publica. 2009;26:209-15.
19. El-Gilany A, Khater M, Gomaa Z, Hussein E, Hamdy I. Psychiatric Disorders among Prisoners: A National Study in Egypt. East Asian Arch Psychiatry. 2016;26:30-8.

20. Vicens E, Tort V, Duenas RM, Muro A, Perez-Arnau F, Arroyo JM, et al. The prevalence of mental disorders in Spanish prisons. Crim Behav Ment Health. 2011;21:321-32.

21. Mundt AP, Alvarado R, Fritsch R, Poblete C, Villagra C, Kastner S, et al. Prevalence rates of mental disorders in Chilean prisons. PloS One. 2013;8:e69109.

22. Dadi AF, Dachew BA, Kisi T, Yigzaw N, Azale T. Anxiety and associated factors among prisoners in North West of Amhara Regional State, Ethiopia. BMC Psychiatry. 2016;31:76-83.

23. Maccio A, Meloni FR, Sisti D, Rocchi MB, Petretto DR, Masala C, et al. Mental disorders in Italian prisoners: results of the REDiMe study. Psychiatry Res. 2015;225:522-30.

24. Barros AJ, Hirakata VN. Alternatives for logistic regression in cross-sectional studies: an empirical comparison of models that directly estimate the prevalence ratio. BMC Med Res Methodol. 2003;3:21.

25. Amorim P. Mini International Neuropsychiatric Interview (MINI): validação de entrevista breve para diagnóstico de transtornos mentais. Rev Bras Psiquiatr. 2000;22:106-15.

26. Ponde MP, Freire AC, Mendonca MS. The prevalence of mental disorders in prisoners in the city of Salvador, Bahia, Brazil. J Forensic Sci. 2011;56:679-82.

27. Silva N, Rosa M, Amboni G, Mina F, Comim C, Quevedo, J. Transtornos psiquiátricos e fatores de risco em uma população carcerária. Arq Catarin Med. 2011;40:72-6.

28. Vargas F, Hoffmeister FX, Prates PF, Vasconcellos SJL. Depressão, ansiedade e psicopatia: um estudo correlacional com indivíduos privados de liberdade. J Bras Psiquiatr. 2015;64(4).

29. Torwane NA, Hongal S, Sahu RN, Saxena V, Chandrashekhar BR. Assessment of prevalence of tobacco consumption among psychiatric inmates residing in Central Jail, Bhopal, Madhya Pradesh, India: A cross-sectional survey. Ind Psychiatry journal. 2013;22:161-4.

30. Tavares GP, Scheffer M, Almeida RMM. Drogas, violência e aspectos emocionais em apenados. Psicol Reflex Crít. 2012;25:89-95.

31. Smolen JR, Araujo EM. Race/skin color and mental health disorders in Brazil: a systematic review of the literature. Cienc Saude Coletiva. 2017;22:4021-30.

32. Battaglia C, di Cagno A, Fiorilli G, Giombini A, Borrione P, Baralla F, et al. Participation in a 9-month selected physical exercise programme enhances psychological well-being in a prison population. Crim Behav Ment Health. 2015;25:343-54.

33. Leijdesdorff S, van Doesum K, Popma A, Klaassen R, van Amelsvoort T. Prevalence of psychopathology in children of parents with mental illness and/or addiction. Current Opinion in Psychiatry. 2017;30(4):312-7.

34. Levitt $\mathrm{L}$, Loper AB. The influence of religious participation on the adjustment of female inmates. Am J Orthopsychiatry. 2009;79:1-7. 